Intussusception risk after rotavirus vaccination in England

Simon B Drysdale ${ }^{1}$, Andrew J Pollard ${ }^{1}$

${ }^{1}$ Oxford Vaccine Group, Department of Paediatrics, University of Oxford, and the NIHR

Oxford Biomedical Research Centre, Level 2, Children's Hospital, Oxford OX3 9DU, United Kingdom

Corresponding author: Dr Simon B Drysdale

Email: simon.drysdale@paediatrics.ox.ac.uk

Tel: +44 (0)1865 231701

Word count (excluding references): 313

Key words: rotavirus vaccine; England; intussusception 


\section{Intussusception risk after rotavirus vaccination in England}

\section{Dear Editor,}

Stowe et al [1] have provided an important analysis of safety of the monovalent rotavirus vaccine (Rotarix ${ }^{\circledR}$, GlaxoSmithKline) in relation to intussusception risk in England. They showed the vaccine causes approximately 21 intussusception admissions annually but prevents almost 25,000 gastrointestinal infection admissions. It, therefore, has a strongly positive risk/benefit profile. Similarly, several previous studies have highlighted a small increased risk of intussusception associated with rotavirus vaccines and ongoing post-marketing surveillance is essential to ensure excellent vaccine safety is maintained.

However, the authors have failed to address the key previous observation that there appears to be an age-related increase in risk of intussusception with dosing later in infancy. This has led to tight recommendations on acceptable timing of administration of rotavirus vaccines. In the UK, two doses are administered between six and 24 weeks of age, with the maximum age for the first dose being 14 weeks and six days, with the second dose at least four weeks after the first dose and before 24 weeks of age [2]. The authors could re-evaluate their analysis with this in mind. In addition, prematurely born infants who received the vaccine while still on the neonatal unit, and subsequently developed intussusception, may not have been captured by the methodology used in this study and potentially represent a high risk group. Current recommendations in England state prematurely born infants should receive the rotavirus vaccine from six weeks chronological age, not corrected for prematurity, if they are clinically stable [2]. If age at vaccination is important it may be that even more 
strict timing recommendations are required to reduce the risk of vaccine-related intussusception. Any further age-restriction would likely result in reduced vaccine coverage, but since several studies [3] have shown that rotavirus vaccine results in significant herd immunity, a small reduction in vaccine coverage may not have a significant impact on the overall vaccine programme effectiveness.

\section{Conflict of interests}

AJP has previously conducted clinical trials of vaccines on behalf of Oxford University funded by vaccine manufacturers but he no longer does so and did not receive any personal reimbursement from them. AJP is chair of the Department of Health's (DH) Joint Committee on Vaccination and Immunisation (JCVI) but the reviews expressed herein do not necessarily represent those of DH or JCVI. SBD has no conflicts of interest to declare.

\section{References}

1 Stowe J, Andrews N, Ladhani S, et al. The risk of intussusception following monovalent rotavirus vaccination in England: A self-controlled case-series evaluation. Vaccine 2016;:6-11. doi:10.1016/j.vaccine.2016.04.050

2 Public Health England. Immunisation against infectious disease 'The Green book'. 2015.

3 Atchison CJ, Stowe J, Andrews N, et al. Rapid declines in age group-specific rotavirus infection and acute gastroenteritis among vaccinated and unvaccinated individuals within 1 year of rotavirus vaccine introduction in England and Wales. J Infect Dis 2016;213:243-9. 